FACTA UNIVERSITATIS (NIŠ)

Ser. Math. Inform. Vol. 36, No 1 (2021), 119-123

https://doi.org/10.22190/FUMI200322010H

Original Scientific Paper

\title{
ON THE AUTOMORPHISMS GROUP OF FINITE POWER GRAPHS
}

\author{
Asma Hamzeh \\ Property and Casualty (Non-life) Insurance Research Group, \\ Insurance Research Center, Tehran, Iran
}

\begin{abstract}
The power graph of a group $G$ is the graph with vertex set $G$, having an edge joining $x$ and $y$ whenever one is a power of the other. The purpose of this paper is to study the automorphism groups of the power graphs of infinite groups.
\end{abstract}

Keywords: Power graphs, infinite groups, automorphism group.

\section{Introduction}

In this paper, we consider only simple undirected graphs. Let $\Gamma$ and $\Delta$ be two graphs. These graphs are said to be isomorphic and write $\Gamma \cong \Delta$, if there exists a bijection $\Phi$ from $V(\Gamma)$ to $V(\Delta)$ such that $u v \in E(\Gamma)$ if and only if $\Phi(u) \Phi(v) \in E(\Delta)$. An isomorphism from $\Gamma$ to itself is called an automorphism of $\Gamma$. We denote the set of all automorphisms on $\Gamma$ as $\operatorname{Aut}(\Gamma)$. A (vertex) coloring of $\Gamma$ is a mapping $c: V(\Gamma) \rightarrow S$, where $S$ is the set of colors. The vertices assigned to a given color form a color class. If $|S|=k$, we say that $c$ is a k-coloring (often we use $S=\{1, \ldots, k\})$. A coloring for $\Gamma$ is proper if adjacent vertices have different colors and it is $k$-colorable if $\Gamma$ has a proper $k$-coloring. The chromatic number $\chi(\Gamma)$ is the least number $k$ such that $\Gamma$ is $k$-colorable. Obviously, $\chi(\Gamma)$ exists as assigning distinct colors to vertices yield a proper $|V(\Gamma)|$-coloring. An optimal coloring of $\Gamma$ is a $\chi(\Gamma)$-coloring and $\Gamma$ is called $k$-chromatic if $\chi(\Gamma)=k$. Finally, $\Gamma$ is planar if it has no subdivision of the $K_{3,3}$ and $K_{5}$.

Suppose $G$ and $H$ are two groups. The group $G$ is called a torsion group if the order of elements of $G$ is finite. The free product $G * H$ of groups $G$ and $H$ is the set of elements of the form $g_{1} h_{1} g_{2} h_{2} \ldots g_{r} h_{r}$, where $g_{i} \in G$ and $h_{i} \in H$, with $g_{1}$ and

Received March 22, 2020; accepted June 22, 2020.

Corresponding Author: Asma Hamzeh, Property and Casualty (Non-life) Insurance Research Group, Insurance Research Center, Tehran, Iran | E-mail: hamze2006@yahoo.com

2010 Mathematics Subject Classification. Primary 05C25; Secondary 05C50.

๑) 2021 by University of Niš, Serbia | Creative Commons License: CC BY-NC-ND 
$h_{r}$ possibly equal to the identity elements of $G$ and $H$. The semidirect product of a group $G$ by a group $H$ is a group $T$ containing $G$ and $H$, where $H$ is normal in $T$ and $G \cap H=\{1\}$ and is denoted $G \rtimes H$. The symmetric group $S_{n}$ of degree $n$ is the group of all permutations on $n$ symbols. The cyclic group of order $n$ is denoted by $Z_{n}$ and for prime number $p$, the Prüfer p-group is denoted by $Z\left(p^{\infty}\right)$.

Graphs associated to algebraic constructions are significant, because they have valuable applications in mathematics and computer science (see, for example, the survey [12] and the monographs [13, 14]). The power graph of a group $G$ is the graph with vertex set $G$, having an edge joining $x$ and $y$ whenever one is a power of the other. The concept of a power graph was first introduced and considered in [11] in the case of groups. Also, they in this paper described the structure of the power graphs of all finite abelian groups. For semigroups, it was first investigated in [10], and then in $[8,9]$. Chakrabarty, Ghosh, and Sen in [4] characterized the class of semigroups $S$ for which $\mathcal{P}(S)$ is connected or complete. As a consequence, they proved that $\mathcal{P}(G)$ is connected for any finite group $G$ and $\mathcal{P}(G)$ is complete if and only if $G$ is a cyclic group of order 1 or $p^{m}$. In [3] Cameron and Ghosh proved that non-isomorphic finite groups may have isomorphic power graphs, but about abelian groups with isomorphic power graphs, the groups are also isomorphic. In [5] Doostabadi, Erfanian, and Jafarzadeh obtained some results on the power graph of infinite groups. The power graphs were also investigated in $[2,17,15]$. In [7], the automorphism groups of the power graph in general for finite groups are computed. Also, Feng et al. [6], computed the full automorphism group of the power graph of a finite group. In [1] Abawajy, Kelarev, and Chowdhury gave a survey of all results on the power graphs of groups and semigroups obtained in the literature.

The purpose of this paper is to study the power graphs of infinite groups and their automorphism groups.

\section{Main Results}

Let $p$ be a fixed prime number. An infinite group $T$ is called a Tarski Monster group for $p$ if every nontrivial subgroup (i.e. Every subgroup other than 1 and $G$ itself) has $p$ elements. The group $G$ is necessarily finitely generated. It is generated by every two non-commuting elements. It is simple. The Tarski groups were first constructed by Olshanskii in 1979. Olshanskii showed in fact that there are continuum-many non-isomorphic Tarski Monster groups for each prime $p>10^{75}$ $[18,19]$.

Let $A$ be an abelian group. The generalized dihedral group $\operatorname{Dih}(A)$ is the semidirect product $A \rtimes Z_{2}$, where $Z_{2}$ is the cyclic group of order 2 , and the generator of $Z_{2}$ maps elements of $A$ to their inverses. If $A$ is cyclic, then $\operatorname{Dih}(A)$ is called a dihedral group. The finite dihedral group $\operatorname{Dih}\left(Z_{n}\right)$ is commonly denoted by $D_{n}$ or $D_{2 n}$. The infinite dihedral group $\operatorname{Dih}(Z)$ is denoted by $D_{\infty}$ and is isomorphic to the free product $Z_{2} * Z_{2}$ of two cyclic groups of order 2 .

First, we state two theorems for the automorphism group of graphs.

Theorem 2.1. Let $\Gamma$ be a graph, then $\operatorname{Aut}(n \Gamma)=\operatorname{Aut}(\Gamma) \imath S_{n}$. 
Theorem 2.2. Let $\Gamma_{1}, \Gamma_{2}, \ldots, \Gamma_{n}$ be distinct graphs and

$$
\Gamma=n_{1} \Gamma_{1} \bigcup n_{2} \Gamma_{2} \bigcup \ldots \bigcup n_{r} \Gamma_{r} .
$$

Then

$$
\operatorname{Aut}(\Gamma)=\left(\operatorname{Aut}\left(\Gamma_{1}\right) \prec S_{n_{1}}\right) \times \ldots \times\left(\operatorname{Aut}\left(\Gamma_{r}\right) \prec S_{n_{r}}\right) .
$$

In the next theorem, we obtain the automorphism group of the power graph of $(Z \times Z \times \ldots \times Z,+)$, where $Z$ is a set of integer numbers.

Theorem 2.3. The automorphism group of the power graph of $(Z \times Z \times \ldots \times Z,+)$ is isomorphic to

$$
\operatorname{Aut}(\mathcal{P}(Z \times Z \times \ldots \times Z,+))=\left(S_{P} \times S_{P} \times \ldots \times S_{P}\right) \text { 乙 } S_{2} .
$$

Where $P$ denotes the set of all prime numbers.

Proof. Consider the group $Z$ under addition. Two integers $a$ and $b$ of $Z$ are adjacent in the power graph if and only if $a \mid b$ or $b \mid a$. This graph has three components containing $\{0\}, Z^{+}$, and $Z^{-}$, where $Z^{+}$and $Z^{-}$are the set of all positive and negative integers, respectively. On the other hand, any integer $n$ is adjacent to all positive multiples of $n$ and there is no edge connecting a positive and a negative number. The components containing all positive and all negative integers is denoted by $H_{1}$ and $H_{2}$ and it can be easily seen that $H_{1} \cong H_{2}$. We now calculate the automorphism group of subgraph $H_{1}$. By power graph structure of $Z$, Aut $\left(H_{1}\right)$ is isomorphic to the automorphism group of the partially ordered set $L=(N, \mid)$, that $N$ is set of the natural numbers. This group is isomorphic to the symmetric group $S_{P}$. Therefore, $\operatorname{Aut}(\mathcal{P}(Z))=S_{P}\left\{S_{2}\right.$.

We now apply induction and Theorems 2.1 and 2.2. Since Aut $(L \times L \times \ldots \times L) \cong$ $S_{P} \times S_{P} \times \ldots \times S_{P}$, so,

$$
\operatorname{Aut}(\mathcal{P}(Z \times Z \times \ldots \times Z,+))=\left(S_{P} \times S_{P} \times \ldots \times S_{P}\right) \imath S_{2} .
$$

This completes the proof.

By [16, Proposition 7], $\mathcal{P}\left(D_{2 n}\right)$ is a union of $\mathcal{P}\left(Z_{n}\right)$ and $n$ copies of $K_{2}$ that share the identity element of $D_{2 n}$ and by [15, Corollary 2.4], the automorphism group of the power graph $D_{2 n}$, if $n$ is a prime power, then is equal to $S_{n-1} \times S_{n}$ and otherwise, is equal to $S_{n} \times \prod_{d \mid \varphi(n)} S_{\varphi(d)}$ that $\varphi$ is Euler's totient function. In the next two theorems, we compute power graphs and automorphism groups of these graphs for generalized dihedral group $\operatorname{Dih}(A)$ and $D_{\infty}$.

Theorem 2.4. For the dihedral groups $\operatorname{Dih}(A)$ and $D_{\infty}$,

$$
\begin{aligned}
\operatorname{Aut}(\mathcal{P}(\operatorname{Dih}(A))) & =\operatorname{Aut}(\mathcal{P}(A)) \times S_{|A|}, \\
\operatorname{Aut}\left(\mathcal{P}\left(D_{\infty}\right)\right) & =\left(S_{P}\left\{S_{2}\right) \times S_{L} .\right.
\end{aligned}
$$

where $L=(N, \mid)$. 
Proof. In the generalized dihedral group $\operatorname{Dih}(A)$, for an abelian group $A$, all elements outside $A$ have order two. So, according to the power graph structure, the power graph of $\operatorname{Dih}(A)$, is the union of a copy of $\mathcal{P}(A)$ and $|A|$ copies of $K_{2}$ that share in identity. So $\operatorname{Aut}(\mathcal{P}(\operatorname{Dih}(A)))=\operatorname{Aut}(\mathcal{P}(A)) \times S_{|A|}$. In this case, if $A$ is a finite abelian group, then the structure of the power graph of it described in [11].

Consider the infinite dihedral group $D_{\infty}=<r, s \mid s^{2}=1, s r s=r^{-1}>$. The power graph of this group is a union of a copy of $\mathcal{P}(Z)$ and infinite copies of $K_{2}$ that share in identity. Thus, by Theorem 2.3 , Aut $\left(\mathcal{P}\left(D_{\infty}\right)\right)=\left(S_{P} \backslash S_{2}\right) \times S_{L}$.

For the sake of completeness, we mention here two important results which are crucial in our investigation of the power graphs of the infinite groups.

Theorem 2.5. [5] Let $G$ be an infinite group. Then $\mathcal{P}(G)$ is complete if and only if $G \cong Z\left(p^{\infty}\right)$ for some prime $p$.

By previous theorem $\operatorname{Aut}\left(Z\left(p^{\infty}\right)\right) \cong S_{N}$.

Theorem 2.6. [5] Let $G$ be a group. Then $\mathcal{P}(G)$ is planar if and only if $G$ is a torsion group and $\pi_{e}(G) \subseteq\{1,2,3,4\}$.

By previous theorem for the Tarski Monster group $T, \mathcal{P}(T)$ is planar.

Theorem 2.7. For the Tarski Monster group $T$, $\operatorname{Aut}(\mathcal{P}(T))=S_{p} \imath S_{N}$. Also $\chi(\mathcal{P}((T))=p$.

Proof. The power graph of the Tarski Monster group $T$ for the prime number $p$ is a union infinite copy of $K_{p}$ that share in identity. So by Theorem 2.1, the automorphism group of this graph is $\operatorname{Aut}(\mathcal{P}(T))=S_{p} 2 S_{N}$. Also, according to power graph structure of the Tarski Monster group, $\chi(\mathcal{P}((T))=p$.

Direct product of graphs $\Gamma_{1}\left(V_{1}, E_{1}\right), \ldots, \Gamma_{n}\left(V_{n}, E_{n}\right)$ is graph $\Gamma(V, E)$, where $V=V_{1} \times \ldots \times V_{n}$ and $E$ is the set of all pairs $\left(\left(a_{1}, \ldots, a_{n}\right),\left(b_{1}, \ldots, b_{n}\right)\right)$ such that $\left(a_{1}, \ldots, a_{n}\right) \neq\left(b_{1}, \ldots, b_{n}\right)$ and $\left(a_{i}, b_{i}\right) \in E_{i} \cup \triangle\left(V_{i}\right)$ for all $1 \leq i \leq n[11]$.

Theorem 2.8. [11] If group $G$ is a direct product of the $p_{i}$-primary components of itself, that $p_{i}$ 's are pairwise distinct primes, then the power graph of $G$ is the direct product of the power graphs of the $p_{i}$-primary component.

Corollary 2.1. We know that $Q / Z=\bigoplus_{p \in P} Z\left(p^{\infty}\right)$ that $Z\left(p^{\infty}\right)$ 's are p-primary components of $Q / Z$. By theorem previous and Theorem 2.5, the power graph of group $Q / Z$ is a direct product of the power graphs of complete graphs.

Acknowledgment. The author would like to thank the referee for the valuable suggestions and comments. 


\section{REFEREN C ES}

1. J. Abawajy, A. V. Kelarev and M. Chowdhury: Power graphs: a survey. Electronic J. Graph Theory and Applications 1 (2013), (2) 125-147.

2. A. R. Ashrafi and B. Soleimani: Normal edge-transitive and 12-arc-transitive Cayley graphs on non-abelian groups of order $2 p q, p>q$ are odd primes. Int. J. Group Theory 5 (2016), 1-8.

3. P. J. CAmeron and S. Ghosh: The power graph of a finite group. Discrete Math. 311 (2011), 1220-1222.

4. I. Chakrabarty, S. Ghosh and M. K. Sen: Undirected power graphs of semigroups. Semigroup Forum 78 (2009), 410-426.

5. A. Doostabadi, A. Erfanian and A. Jafarzadeh: Some results on the power graphs of finite groups. Science Asia 41 (2015), 73-78.

6. M. FEnG, X. MA and K. WANG: The full automorphism group of the power (di)graph of a finite group. European J. Combin. 52 (2016), 197-206.

7. A. HAMzeH and A. R. AshrAFi: Automorphism group of supergraphs of the power graph of a finite group. European J. Combin. 60 (2017), 82-88.

8. A. V. Kelarev and S. J. QuinN: A combinatorial property and power graphs of semigroups. Comment. Math. Univ. Carolin. 45 (1) (2004), 1-7.

9. A. V. Kelarev and S. J. Quinn: Directed graphs and combinatorial properties of semigroups. J. Algebra 251 (1) (2002), 16-26.

10. A. V. Kelarev, S. J. Quinn and R. Smolíková: Power graphs and semigroups of matrices. Bull. Austral. Math. Soc. 63 (2) (2001), 341-344.

11. A. V. Kelarev and S. J. Quinn: A combinatorial property and power graphs of groups. Contributions to General Algebra 12 (Vienna, 1999), 229-235, Heyn, Klagenfurt, 2000.

12. A. V. Kelarev, J. Ryan and J. Yearwood: Cayley graphs as classifiers for data mining: the in uence of asymmetries. Discrete Math. 309 (2009), 5360-5369.

13. A. V. Kelarev: Ring constructions and applications, World Scientific, NJ, 2002.

14. A. V. Kelarev: Graph algebras and automata, Marcel Dekker, New York, 2003.

15. Z. Mehranian, A. Gholami and A. R. Ashrafi: A note on the power graph of a finite group. Int. J. Group Theory 5 (1) (2016), 1-10.

16. M. Mirzargar, A. R. Ashrafi and M. J. Nadjafi-Arani: On the power graph of a finite group. Filomat 26 (2012), 1201-1208.

17. M. Shaker and M. Ali Iranmanesh: On groups with specified quotient power graphs. Int. J. Group Theory 5 (2016), 49-60.

18. A. Yu. OlshanskiI: An infinite group with subgroups of prime orders. Math. USSR Izv. 16 (1981), 279-289; translation of Izvestia Akad. Nauk SSSR Ser. Matem. 44 (1980), 309-321.

19. A. Yu. OlshanskiI: Groups of bounded period with subgroups of prime order. Algebra and Logic 21 (1983), 369-418; translation of Algebra i Logika 21 (1982), 553-618. 